Physical signs

\section{Some neurological signs}

\section{G D Perkin}

\section{A series looking at aspects of clinical examination}

A series devoted to the value of particular physical signs in neurological practice needs no apology. Though one suspects that succeeding generations of neurologists have bemoaned the lack of clinical expertise among their juniors there can be no doubt that the remarkable advance in neuroimaging and allied techniques that has been seen over the past 20 years has altered the delicate balance between clinical appraisal and investigation. An overreliance on sophisticated investigative techniques has a number of potential pitfalls. The interpretation of those investigations remains a highly skilled process and is prone to error. Some of these techniques are of such sophistication that abnormalities are frequently discovered that bear no relation to the clinical problem. Without an appropriate level of history taking and examination, such abnormalities may be misinterpreted as being relevant to the patient's presentation. Indeed there remains as much skill in the decision not to investigate a particular patient as to do so. That decision rests at least in part on the ability to perform an adequate neurological examination and to be able to distinguish the normal from the abnormal.
This series will look at nine aspects of the clinical examination that I felt merited discussion regarding their value in determining diagnosis or, at least, in the localisation of a neurological disorder. The list is subjective and to some extent perhaps arbitrary but I believe it embraces a number of areas where correct interpretation of an abnormal finding is of major value to the clinician in patient assessment. Individual contributors therefore have been asked where possible to look at issues such as specificity and sensitivity when discussing a particular physical sign. The series I trust will prove both informative and stimulating.

J Neurol Neurosurg Psychiatry 2002;73:1 10

\section{Authors' affiliations}

G D Perkin, West London Neurosciences Centre, Charing Cross Hospital, Fulham Palace Road, London W6 8RF, UK

Correspondence to: Dr G D Perkin; d.perkin@ic.ac.uk

\section{EDITORIAL COMMENTARIES}

Multiple sclerosis

\title{
Neutralising antibodies to the beta interferons
}

\section{A J Coles}

\section{Determining and preventing neutralising antibodies}

$\mathrm{T}$ he amazing ability of the immune system to make fine distinctions between self and non-self is critical to its function-but a problem for treatments that mimic natural proteins, such as recombinant human factor VIII, whose use is significantly compromised by neutralising antibodies. So too is interferon beta treatment of multiple sclerosis. Antibodies against the beta interferons have usually ${ }^{2}$ but not always, ${ }^{3}$ been associated with increased clinical and magnetic resonance imaging markers of disease activity. Therefore, the clinician needs to know how common such antibodies are, how reliably they are detected, what to do if they are discovered, and how to prevent them. Answers to some of these questions come from an Italian study in this issue $(\text { pp 148-53) })^{4}$ of 125 patients on the three available interferon beta products.

Unsurprisingly, Bertoletto et al found that Avonex, an interferon beta la, generated fewer antibody responses than interferon beta $\mathrm{lb}$ (Betaferon), which is more "foreign" to the immune system. Interferon beta la is produced by mammalian cells and is identical to the natural cytokine in sequence and glycosylation, whereas interferon beta $\mathrm{lb}$ has two sequence differences (des-Met-1 and Cys17Ser) and, being produced in Escherichia coli, is not glycosylated. Rebif, the other interferon beta la, induced fewer antibodies than Betaferon but more than Avonex. Although these differences were not significant in Bertoletto et al's series, they reflect a ranking that is emerging clearly from the literature, especially from the largest study on 754 patients in Denmark. ${ }^{5}$ Rebif probably provokes more antibodies than Avonex, despite being chemically identical, because the immune system finds Rebif's delivery route and dosing (22-44 $\mathrm{mg}$ in three weekly subcutaneous doses) more "unnatural" than that of Avonex (30 mg in one weekly intramuscular dose).

The prevalence of antibodies against interferon beta is confused in the literature, ranging from $28-80 \%$ of patients treated with Betaferon. In part this may reflect when patients were tested. Bertoletto et al found that neutralising antibodies may be transient and may appear at any time from 3 to 18 months after starting treatment. But also there are technical issues. Binding assays such as enzyme linked immunosorbent assays (ELISAs) are relatively easy to standardise but do not distinguish antibodies that impair interferon beta function ("neutralising" antibodies) from those that do not. Bioassays can discriminate these but they are difficult to compare across centres. Bertoletto et al used the most popular: patient's serum was tested for its ability to prevent interferon beta inhibiting encephalomyocarditis virus from killing a human lung carcinoma cell line. They reported the prevalence of persistent neutralising antibodies, after 18 months of treatment, as $32 \%$ in patients who received Betaferon, 19\% for Rebif, and $4 \%$ for Avonex. The thresholds for positivity in such studies are arbitrary and have not been validated against clinical data.

Once a patient has persistent neutralising antibodies, switching to another interferon beta is illogical, as the antibodies cross react. Nor would a "drug holiday" help, as the immune system has a long memory. Strategies to prevent neutralising antibodies may include 
using a tolerising drug to initiate treatment, concomitant immunosuppression, mucosal routes of administration, and either much higher or lower doses.

J Neurol Neurosurg Psychiatry 2002;73:110-111

Authors' affiliations

A J Coles, Department of Neurology, Box 165, Addenbrooke's Hospital, Hills Road, Cambridge CB2 2QQ, UK

Correspondence to: Dr A Coles; ajc1020@medschl.cam.ac.uk
Conflict of interest: AC has received a fee for speaking at a conference organised by Serono.

\section{REFERENCES \\ 1 Duquette $\mathbf{P}$, Girard M, Dubois R, et al. Neutralizing antibodies during treatment of multiple sclerosis with interferon beta- $1 \mathrm{~b}$ : experience during the first three years. The IFNB Multiple Sclerosis Study Group and the University of British Columbia MS/MRI Analysis Group. Neurology 1996;47:889-94. \\ 2 Francis G, Hughes R, King J, et al. PRISMS-4: Long-term efficacy of interferon-beta-la in relapsing MS. Neurology 2001;56:1628-36. \\ 3 Durelli L, Verdun E, Barbero P, et al. Every-other-day interferon beta-1 $\mathrm{b}$ versus}

once-weekly interferon beta-la for multiple sclerosis: results of a 2-year prospective randomised multicentre study (INCOMIN). Lancet 2002;359: 1453-60.

4 Bertolotto A, Malucchi S, Sala A, et al. Differential effects of three interferon betas on neutralising antibodies in multiple sclerosis patients: a follow up study in an independen laboratory. I Neurol Neurosurg Psychiatry 2002; 73: 148-53.

5 Ross C, Clemmesen KM, Svenson M, et al. Immunogenicity of interferon-beta in multiple sclerosis patients: influence of preparation, dosage, dose frequency, and route of administration. Danish Multiple Sclerosis Study Group. Ann Neurol 2000;48:706-12.

\section{Functional reorganisation of memory following traumatic brain injury: a study with $\mathrm{H}_{2}{ }^{15} \mathrm{O}$ PET}

\section{J T E Richardson}

\section{Neuroimaging holds future promise for before and after comparisons}

$\mathrm{T}$ raumatic brain injury (TBI) is a major cause of deaths following accidents and a major cause of disablement and morbidity among the survivors. The clinical problem of treating this vast number of patients is not going to be solved by a dramatic medical breakthrough. Rather, it must be tackled by seeking a better appreciation of the condition that these people present. The paper by Levine et al (this issue, pp 173$81)^{1}$ adds to the growing body of studies in which neuroimaging techniques have been used to study long term changes in survivors of moderate or severe TBI.

Levine et al carried out PET in six patients who had sustained TBI roughly four years previously and in 11 healthy controls. Separate scans were obtained during the encoding and retrieval phases of a simple cued recall task and attention was focused on the differences in activation between the two phases. The individual findings varied with focal lesions. However, as a group they had more widespread activation during the retrieval phase than the controls, with reduced activation in areas involved in normal memory retrieval. This was regarded as evidence for neural reorganisation due to diffuse axonal injury.
This study combined the application of neuroimaging technology with an understanding of the cognitive mechanisms involved in the relevant experimental task. Levine et al also paid due attention to both between group variation and within group variation. They considered but rejected the idea that these changes might just have been an artefact of impaired performance. Indeed, the same pattern was apparent in three patients whose retrieval was at a normal level, although some aspects were seen only in the three patients with poor performance.

Two issues need to be addressed in further research. Firstly, would similar changes be seen in patients with mild rather than severe TBI? The prevalence of mild TBI is many times greater than that of severe TBI, and many suspect that mild TBI may give rise to persistent yet subtle deficits even in patients who otherwise make a good functional recovery. ${ }^{2}$ of course, such deficits may have predated the TBI and were not caused by it. ${ }^{3}$ This problem is inherent in any study that compares groups defined on the basis of their clinical history.

So a second issue raised by this study is this: are the apparent differences between patients with TBI causally related to their injuries? Ideally, one would like to have premorbid evidence from the people in question. Of course, the number of people who have undergone any specific PET procedure before sustaining a TBI is vanishingly small, but other evidence may be available. Bigler and Snyder ${ }^{4}$ compared computed tomography with magnetic resonance imaging carried out in four people before and after they sustained mild TBI. The authors failed to find any gross differences but more detailed analyses might have revealed clinically significant changes. With the increasingly widespread use of neuroimaging techniques, comparisons of this sort should be entirely feasible in the future.

J Neurol Neurosurg Psychiatry 2002;73:111

\section{Author's affiliation}

J T E Richardson, Institute of Educational Technology, The Open University, Walton Hall, Milton Keynes MK7 6AA, UK

Correspondence to: Professor J T E Richardson; i.t.e.richardson@open.ac.uk

\section{REFERENCES}

1 Levine B, Cabeza R, McIntosh AR, et al. Functional reorganisation of memory after traumatic brain injury: a study with $\mathrm{H}_{2}{ }^{15} \mathrm{O}$ positron emission tomography. J Neurol Neurosurg Psychiatry 2002;73:173-81.

2 Marschark M, Richtsmeier LM, Richardson JTE, et al. Intellectual and emotional functioning in college students following mild traumatic brain injury in childhood and adolescence. J Head Trauma Rehabil 2000; 15: 1227-45

3 Ponsford J, Willmott C, Rothwell A, et al Cognitive and behavioral outcome following mild traumatic head injury in children. $J$ Head Trauma Rehabil 1999;14:360-72.

4 Bigler ED, Snyder JL. Neuropsychological outcome and quantitative neuroimaging in mild head injury. Arch Clin Neuropsychol 1995; 10:159-74. 


\section{Coffee and subarachnoid haemorrhage}

\section{W T Longstreth Jr}

\section{The link between coffee and subarachnoid haemorrhage is unresolved}

Y ou may be making enemies, especially in Seattle, if you conclude from the study of Isaksen et al that coffee is a risk factor for subarachnoid haemorrhage (this issue, pp 185-7). ${ }^{1}$ Love of java necessitates a critical evaluation. Are we dealing with coincidence, confounding, or causation? In a casecontrol study, these investigators drew subjects from a population based health survey of inhabitants in the municipality of Tromsø, Norway. At variable times before the bleeding (maximum 186 months) participants had been evaluated. The investigators found that cigarette smoking and high systolic blood pressure increased risk, as have others. ${ }^{2}$ The trend was for high cholesterol to reduce risk but not significantly, perhaps reflecting the small number of participants $(n=26)$. Drinking six or more cups of coffee per day yielded an odds ratio of 3.86 even after controlling for these other factors in multivariable analyses.

Is the association a coincidence? Few have examined coffee as a risk factor for stroke, ${ }^{3}$ let alone subarachnoid haemorrhage, so we cannot look for consistency with previous studies. The investigators did not specify how many candidate risk factors they examined, but the more examined, the greater the risk that a significant finding will emerge by chance alone. Perhaps the association is real but not causal. The search for risk factors is important because, if associations are causal, the possibility exists of understanding better pathophysiology and prevention. Rather than being causal, could the association with coffee have been confounded by other factors related both to coffee consumption and to subarachnoid haemorrhage, such as alcohol consumption? Perhaps the association would have disappeared after controlling for alcohol consumption. Also by examining different doses of coffee, as they did for cigarette smoking, the investigators could have strengthened their argument for a causal association.

If the association were real, how might coffee increase the risk of subarachnoid haemorrhage? Bleeding is typically the culmination of aneurysm formation and rupture. Examining coffee as a risk factor in patients with unruptured intracranial aneurysms or in patients with multiple aneurysms may address questions of formation. How the association varied as a function of the time since the onset of the bleeding may address questions of rupture. Caffeine can cause an increase in blood pressure, ${ }^{4}$ perhaps putting those who harbour an intracranial aneurysm and who drink six cups or more of caffeinated coffee per day at increased risk for rupture compared with those who drink less or do not drink coffee at all. In the current study we do not know whether the coffee was caffeinated and whether other caffeinated beverages, such as tea and cola drinks, were examined. Questions about rupture would require knowledge about the use of the beverage in the time immediately before the onset of the bleeding, not months before. As is so often the case with such unexpected findings, more studies are needed before we can judge the importance of this intriguing and novel observation, especially about such a common exposure. So for now sip your coffee but with some lingering concern about this unresolved issue.

J Neurol Neurosurg Psychiatry 2002;73:112

\section{Authors' affiliations}

W T Longstreth Jr, Department of Neurology, University of Washington, Harborview Medical Center, 325 Ninth Avenue, Seattle, Washington 98104-2420, USA

Correspondence to: Dr W T Longstreth; wl@u.washington.edu

\section{REFERENCES}

1 Isaksen J, Egge A, Waterloo K, et al. Risk factors for aneurysmal subarachnoid haemorrhage: the Tromsø study. I Neurol Neurosurg Psychiatry 2002;73: 185-7.

2 Teunissen LL, Rinkel GJ, Algra A, et al. Risk factors for subarachnoid hemorrhage: a systematic review. Stroke 1996:27:544-9.

3 Hakim AA, Ross GW, Curb JD, et al. Coffee consumption in hypertensive men in older middle-age and the risk of stroke: the Honolulu heart program. J Clin Epidemiol 1998:51:487-94.

4 James JE. Is habitual caffeine use a preventable cardiovascular risk factor? Lancet 1997;349:279-81 